\title{
Fuzzy PSS Design for a Multi-machine Power System Using Improved Genetic Algorithm
}

\author{
H. Shayeghi ${ }^{1, *}$, H. A. Shayanfar ${ }^{2}$, O. Abedinia ${ }^{3}$ \\ ${ }^{1}$ Technical Engineering Department, University of Mohaghegh Ardabili, Ardabil, Iran \\ ${ }^{2}$ Electrical Engineering Department, Iran University of Science and Technology, Tehran, Iran \\ ${ }^{3}$ Electrical Engineering Department, Islamic Azad University, Science and Research Branch, Tehran, Iran
}

\begin{abstract}
This paper addresses a robust fuzzy controller to damp low frequency oscillation following disturbances in power systems. In this research the fuzzy controller is used as a Power System Stabilizer (PSS) to improve the stability in power system. The rule base of the proposed PSS is optimized offline automatically by the improved Genetic Algorithm (GA). Usually in a rule base fuzzy control system, exact tuning of fuzzy rule base is very important for achieving the desired level of robust performance. Hence, the rule set of fuzzy PID based stabilizer is tuned by GA to reduce fuzzy system effort and find optimal stabilizer. The effectiveness of the proposed method is tested on the New England 10-machine 39-bus standard power system and compared with the Tabu Search based tuned PSS under different loading condition through ITAE and FD performance indices.
\end{abstract}

Keywords PSS, Fuzzy Controller, GA, Multi-machine Power Systems, Power System Dynamics

\section{Introduction}

In early sixties, most of the generators were getting interconnected and the automatic voltage regulators (AVRs) were more efficient. With bulk power transfer on long and weak transmission lines and application of high gain, fast acting AVRs, small oscillations of even lower frequencies were observed. The stability of the system, in principle, can be enhanced substantially by application of some form of close-loop feedback control. Over the years a considerable amount of effort has been extended in laboratory research and on-site studies for designing such controllers. The problem, when first encountered, was solved by fitting the generators with a feedback controller which sensed the rotor slip or change in terminal power of the generator and fed it back at the AVR reference input with proper phase lead and magnitude so as to generate an additional damping torque on the rotor[1]. Damping power oscillations using supplementary controls through turbine, governor loop had limited success. With the advent fast valving technique, there is some renewed interest in this type of control[2]. This device came to be known as a Power System Stabilizer (PSS).

Over the years, a number of techniques have been developed for designing PSSs. Conventionally; lead-lag controller has been widely used in power system control to damping the low frequency oscillations. In order to determine the

* Corresponding author:

hshayeghi@gmail.com (H. Shayeghi)

Published online at http://journal.sapub.org/computer

Copyright (C) 2012 Scientific \& Academic Publishing. All Rights Reserved coefficient of lead-lag controller, several control algorithms based on conventional methods are proposed[3]. Conventional Power System Stabilizers (CPSSs) are designed based on linear models representing the system's generators operating at a certain operating point[3]. The performance of these designed CPSSs is acceptable as long as the system is operating close to the operating point for which the system model is obtained. However, CPSSs are not able to provide satisfactory performance results over wider ranges of operating conditions. Considerable efforts have been directed towards developing adaptive PSS[3-5]. The basic idea behind adaptive techniques is to estimate the uncertainties in the plant on-line based on measured signals. However, adaptive PSSs can only deal with systems of known structure. Furthermore, adaptive controllers cannot use human experience which is expressed in linguistic descriptions. This problem is overcome by using artificial intelligence (fuzzy logic, neural networks, decision trees)- based techniques for the design of PSSs.

The main problem encountered in the conventional PSS design is that the power system constantly experience changes in operating conditions due to variation in generation and load patterns, as well as change in transmission networks. Thus, the investigation carried out using this approaches reveal that it exhibits poor dynamic performance. To overcome these difficulties of PSS design, intelligent optimization based techniques have been introduced[4]. Artificial Neural Network (ANN) is an intelligent method which is used for PSS tuning[5]. This technique has its own advantages and disadvantages. The performance of power system is improved by ANN based controller but, the main 
problem of these controllers are the long training time and selecting the number of layers and number of neurons in each layers[6].

Fuzzy logic controllers are exhibited excellent control performance by utilizing experimental knowledge on the plant characteristic when the plant dynamics is uncertain or quite complex so that analytical control laws cannot be derived from it. They have been successfully used to replace the conventional controllers in many industrial control situations. Recently, fuzzy based PSS schemes have been proposed[7-9]. Unlike other classical control methods, fuzzy logic are model-free controller; i.e. it does not require an exact mathematical model of the controlled system. Moreover, speed and robustness are the most significant properties in comparison to other classical schemes. However, subjective choice of the fuzzy controller's parameters does not guarantee optimal performance of the system.

Successful design of a fuzzy control system depends on several factors such as choice of the rule set, membership functions, inference mechanism and the defuzzification strategy. Of these factors, selection of an appropriate rule set is more difficult because, it is a computationally expensive combinatorial optimization problem. Sometimes for fuzzy controllers, rule base is derived from human expert who have acquired their knowledge through experience. However, experts may not always be available even when available extraction of an appropriate set of rules from the expert may be tedious, time consuming and process specific. Thus, extraction of an appropriate set of rules or selection of an optimal set of rules from the set of possible rules is an important and essential step toward the design of any successful fuzzy logic controllers. Hence, in this paper, a Genetic Algorithms (GA) based rule generation method is automatically proposed for fuzzy PSS design to improve power system stability and reduce the design effort. Genetic algorithm is a strong optimization technique which is independent on the complexity of problems and the prior knowledge is not available. In addition, the proposed fitness function is very simple which tries to ensure that controller operates over the entire input space with a good settling time[4,10-11]. In this study, the Fuzzy PID (FPID) controller is constructed as a set of control rules and then gains of PID controller are tuned online from the knowledge base and the fuzzy inference. This newly developed control strategy combines the advantage of GAs and fuzzy control techniques and leads to a flexible controller with simple structure that is easy to implement.

The New England 10-39 buses power system, under various system configurations and loading conditions is employed to illustrate the effectiveness of the proposed method in comparison with the robust Tabu Search based tuned PSS (TSPSS)[12] through nonlinear time simulation and some performance indices. Results evaluation show that the proposed method is effective and alternative to conventional fixed gain stabilizer design as it retains the simplicity of the conventional PSS and still guarantees a robust acceptable performance over a wide range of operating and system condition.

\section{Fuzzy PID Controller Design}

Nowadays, fuzzy theory is used in almost all sectors of industry and science. One of the applications is in power system control. Fuzzy logic control is one of the most successful areas in the application of fuzzy theory and is an excellent alternatives to the conventional control methodology when the processes are too complex for the analysis run by conventional mathematical techniques[13-14]. Because of the complexity and multi-variable conditions of the power system, conventional control methods may not give satisfactory solutions. On the other hand, their robustness and reliability make fuzzy controllers useful for solving a wide range of control problems in power systems. In general, the application of the fuzzy logic method to PID control design in the PSS design can be classified in two major categories according to the way of their construction[15]:

1. A typical PSS is constructed as a set of heuristic control rules, and the control signal is directly deduced from the knowledge base.

2. The gains of the conventional PID controller are tuned on-line in terms of the knowledge basis and fuzzy inference, and then, the conventional PID controller generates the control signal.

Fig. 1 shows the block diagram of the classical fuzzy type controller to PSS design for each generator.

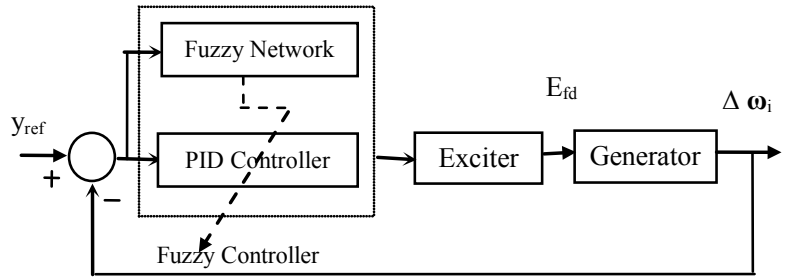

Figure 1. The classical FPID controller design problem

For designing fuzzy logic controller, there are five parts involved in the fuzzy inference process:

1- Fuzzification of the input variables.

2- Application of the fuzzy operator (AND or OR) in the anteceden.

3- Implication from the antecedent to the consequent.

4- Aggregation of the consequents across the rules.

5- Defuzzification.

The structure of the classical FPID controller has twolevel controller. The first level is fuzzy network and the second level is the PID controller. The controller block is formed by fuzzification of $\left(\Delta \omega_{i}\right)$, the interface mechanism and defuzzification. Therefore, $u_{i}$ is a control signal that applies to the excitation system in each generator. By taking $\Delta \omega_{i}$ as the system output, control vector for the conventional PID controller is given by:

$$
u_{i}=K_{P i i} \Delta \omega_{i}(t)+K_{I i} \int_{0}^{t} \Delta \omega_{i}(t) d t+K_{d i} \Delta \dot{\omega}_{i}(t)
$$

The parameters, $K_{I i}, K_{d i}$ and $K_{p i}$ are determined by a set of fuzzy rules of the form: 
If $\Delta \omega_{\mathrm{i}}$ is $\mathrm{A}_{\mathrm{i}}$ and $\Delta\left(\Delta \omega_{\mathrm{i}}\right)$ is $B_{i}$ then $K_{d i}$ is $C_{i}$ and $K_{p i}$ is $D_{i}$ and $K_{I i}$ is $E_{i}, i=1,2, \cdots, n$.

Where, $A_{i}, B_{i,} C_{i}, D_{i}$ and $E_{i}$ are fuzzy sets on the corresponding supporting sets.

In many cases, performance of FPID controller depends on a designed knowledge base in which fuzzy control rules are defined[16]. In traditional method, the rule base is determined by experience and control knowledge of human expert. However, it is a trial and error process and takes a long time and quite significant cost. Thus, the automatic design method for FPID control, which can generate an optimal rule tables without human experts, is desirable. In this study, GAs technique is utilized to find optimal rule sets of the FPID based PSS.

\section{GA-based Fuzzy PSS Design}

GAs are search algorithms based on the mechanism of natural selection and natural genetics that operate without knowledge of the task domain and utilize only the fitness of the evaluated individuals. They can be considered as a general-purpose optimization method and have been successfully applied to search and optimization[17,14-15]. During evolution, GAs requires only information the quality of the fitness value produced by each parameter set. This differs from many optimization methods requiring derivative information or complete knowledge of the problem structure and parameter. Hence, the GA is more suitable to deal with the problem of lacking experience or knowledge than other searching methods in particular, when the phenomena being analyzed are describable in terms of the rules for action and learning processes[18-19].

In this paper, a GA-based Fuzzy (GAF) controller is proposed to design of PSS that synthesize the advantage of the GAs and fuzzy control techniques to achieve adaptable robust performance. The motivation of using the proposed GAF based PSS is to reduce the fuzzy system effort and determine the optimal controller scheme such that the relative stability is guaranteed and the time domain specifications concurrently secured.

Actually obtaining the optimal decision-making logic for the proposed GAF control strategy to achieve the desired level of system robust performance is really important, because it is a computationally expensive combinatorial optimization problem. Usually, the rule base sets are determined by experience and control knowledge of human expert. But, expert may not always be available even where available it is a trial-error process and takes much time and cost. Thus, to overcome these drawbacks and reduce fuzzy system effort and cost, a modified GA technique is being used to construct the optimal rule base sets of the proposed fuzzy type PID PSS. Figure 2 shows the structure of the proposed GAF PSS to improve power system stability.

In the proposed rule base optimization problem, the membership function sets for the $\mathrm{K}_{\mathrm{Pi}}, \mathrm{K}_{\mathrm{Ii}}$ and $\mathrm{K}_{\mathrm{di}}$ are defined as triangular partitions with five segments from 0 to 1 as shown in Fig. 3. Zero (ZO) is the center membership function. The remaining parts of the partition are Negative Big (NB), Negative Small (NS), Positive Small (PS) and Positive $\mathrm{Big}(\mathrm{PB})$. The membership function sets for $\Delta \omega \mathrm{i}, \Delta(\Delta \omega \mathrm{i})$ is the same as Membership Function (MF) sets as shown in Fig. 3.

\subsection{Optimization Rule Base}

In the proposed PSS, construction of the rule Tables are difficult to contribute according to human experience and knowledge. To acquire an optimal combination, we adapt GAs as the search method. In this work, the GA module works offline. Among all the various GAs, classical GA is the simplest one without loss of efficiency. In this study, we adopted a modified GA to improve the speed of the convergence and find the optimum value of fitness function. Fig. 4 shows the flowchart of the modified GA approach for the optimization. Before proceeding with the GA approach, there are two preliminaries to be finished.

1) Definition of suitable coding: coding is one of the most attractive problems in the GA's solution space. According to Fig. 3, each rule Table has 20 rules. Also, the fuzzy set $\{\mathrm{NB}$, $\mathrm{NZ}, \mathrm{Z}, \mathrm{PS}, \mathrm{PB}\}$ can be represented with an integer set $\{1,2$, $3,4,5\}$. Thus, the order of parameters is coded into the chromosome (individual). A chromosome represents a candidate solution of the problem.



Figure 2. Structure of the proposed GA-F based PSS 


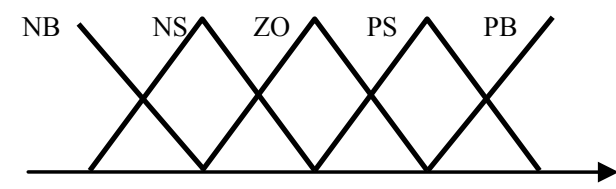

Figure 3. The MF sets for $K_{P i}, K_{l i}$ and $K_{d i}$

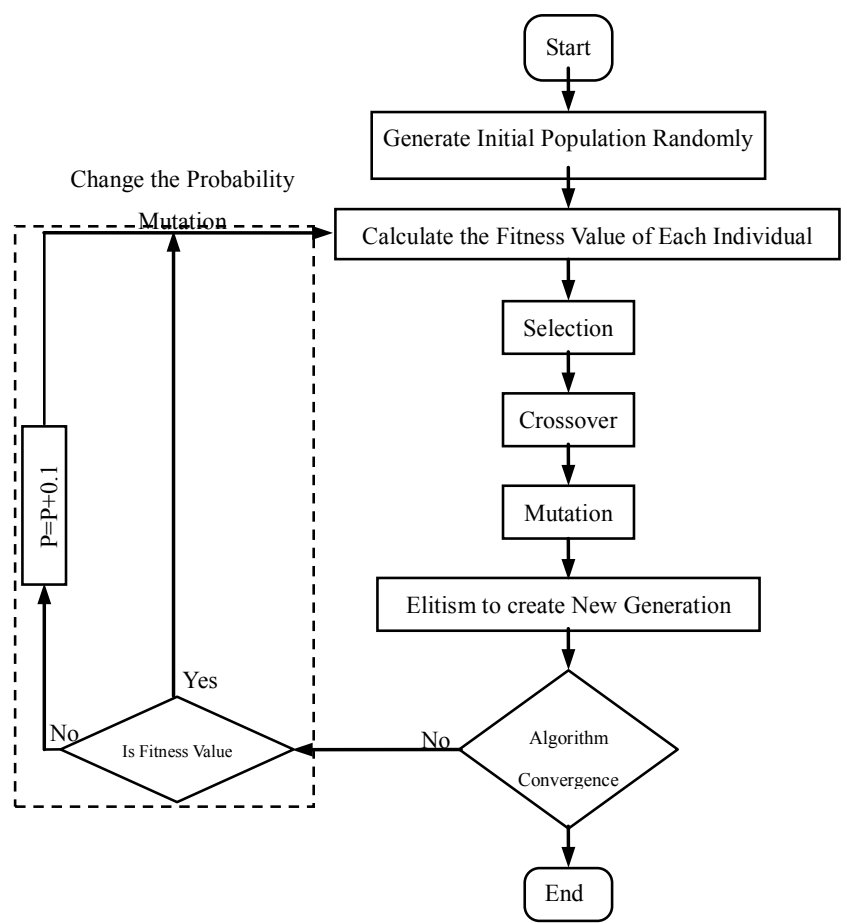

Figure 4. Modified GA approach to find optimal rule base sets

In this method, a solution candidate is expressed by binary coding. Consequently, number of rules and fuzzy set for the $K_{P i}, K_{I i}$ and $K_{d i}$ in each generation are expressed in term of string consisting of 0 and 1 as shown in Fig. 5.

2) Choice of fitness functions: the second primary to be finished is choosing the problem-dependent fitness function. In this study, trend of the Figure of Demerit (FD) and Integral of the Time multiplied Absolute value of the Error (ITAE) based function is an alternative of the conventional maximization of fitness function, which defined as equation (2)[15].

$$
f(I T A E)=\frac{1}{1+M S E(I T A E)}
$$

Where,

$$
\operatorname{MSE}(\operatorname{ITAE})=\left(\sqrt{\sum_{i=1}^{m} I T A E_{i}}\right) / m, \operatorname{ITAE} E_{i}=100 \int_{0}^{10} t\left(\left|\Delta \omega_{i}\right|\right) d t
$$

After deciding these two preliminaries, we should choose the genetic operators. This algorithm consists of elitism selection and three kinds of genetic operators which are selection, crossover and mutation to create new generation.

1) Selection: selection chooses the individuals in the population as parent individuals to create offspring for the next generation, whose purpose is to emphasize the fitter individuals in the population in hopes that their offspring will in turn have even higher fitness. In this work, the roulette wheel selection is adopted.

2) Crossover: Instead of the single-point crossover, we adopt the two-point crossover. For example, the parent individuals $h_{1}$ and $h_{2}$ given to be crossover at the points $k$ and $l$ with the crossover probability $P_{c}$. Results in the new offspring $h^{\prime}{ }_{1}$ and $h^{\prime}{ }_{2}$ are expressed as:

$$
\begin{aligned}
& h_{1}^{\prime}= \begin{cases}h_{2 i} & k<i<l \\
h_{l i} & \text { otherwise }\end{cases} \\
& h_{2}^{\prime}= \begin{cases}h_{1 i} & k<i<l \\
h_{2 i} & \text { otherwise }\end{cases}
\end{aligned}
$$

3) Mutation: A position of each gene with probability $P_{m}$ which is a possible candidate for the mutation is selected randomly and then value of gene, 0 or 1 , changed to, 1 or 0 , respectively.

4) Elitism: Elitism guarantees that the best string individual survives until the last generation. Among parents and their children that are generated by crossover and mutation individuals, they have the best fitness function only survive to the next generation. Size of the individuals in the next generation is the same as the initial population size.

The genetic algorithms are powerful search techniques to optimization, but some well-known disadvantage in GA are poor convergence of the classical GA near the global optimum and convergence to the suboptimum. In order to overcome these drawbacks, the following procedure is being used in the proposed modified GA:

In each iteration, probability of the mutation $\left(\mathrm{P}_{\mathrm{m}}\right)$ is changed according to Fig. 4 if the fitness function value does not improve in comparison with the previous generation. This method guarantees algorithm convergence to the near optimum solution.

\section{Case Study}

In this study, the 10-machine 39-bus power system shown in Fig. 6 is considered. Furthermore operating of system is tested in three different conditions as nominal, lightly and heavily loading conditions. Details of the system data and operating condition are given in Ref.[12].

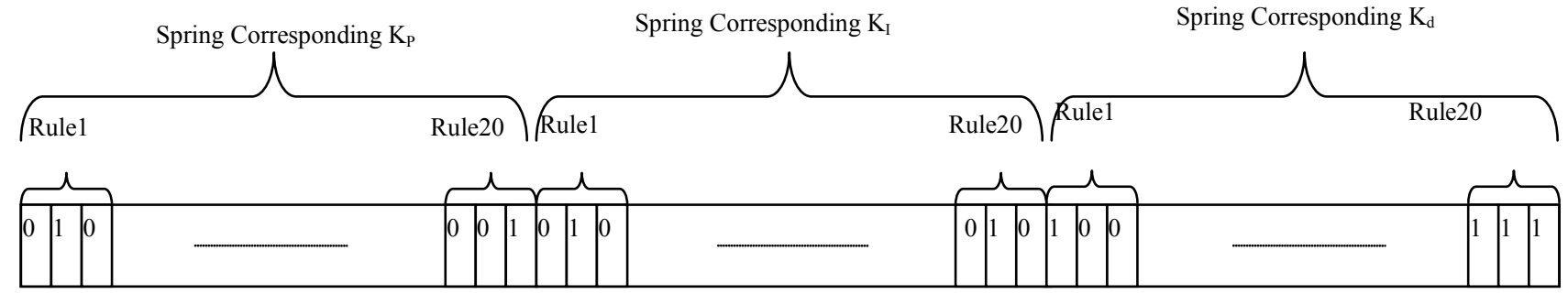

Figure 5. String encoding the proposed fuzzy control rule Table 


\subsection{GA-based Fuzzy PSS Design}

The proposed fuzzy PSS is connected to machines G5, G7 and G9 in the test system. Here, the modified GA evolution procedure is applied to produce rule Tables of the proposed GAF based PSS to guarantee relative stability and concurrently secure the time domain specifications. In this work, these parameters are listed in Table 1. The plot of obtained fitness function value is shown in Fig. 7. The Results of fuzzy rule base sets are listed in Tables 2-4.

Table 1. GA set parameters

\begin{tabular}{|c|c|}
\hline Number of generation & 90 \\
\hline Population size & 20 \\
\hline Crossover rate & 0.97 \\
\hline Mutation rate & 0.08 \\
\hline
\end{tabular}

Table 2. Optimal rule base of $\mathrm{K}_{\mathrm{pi}}$

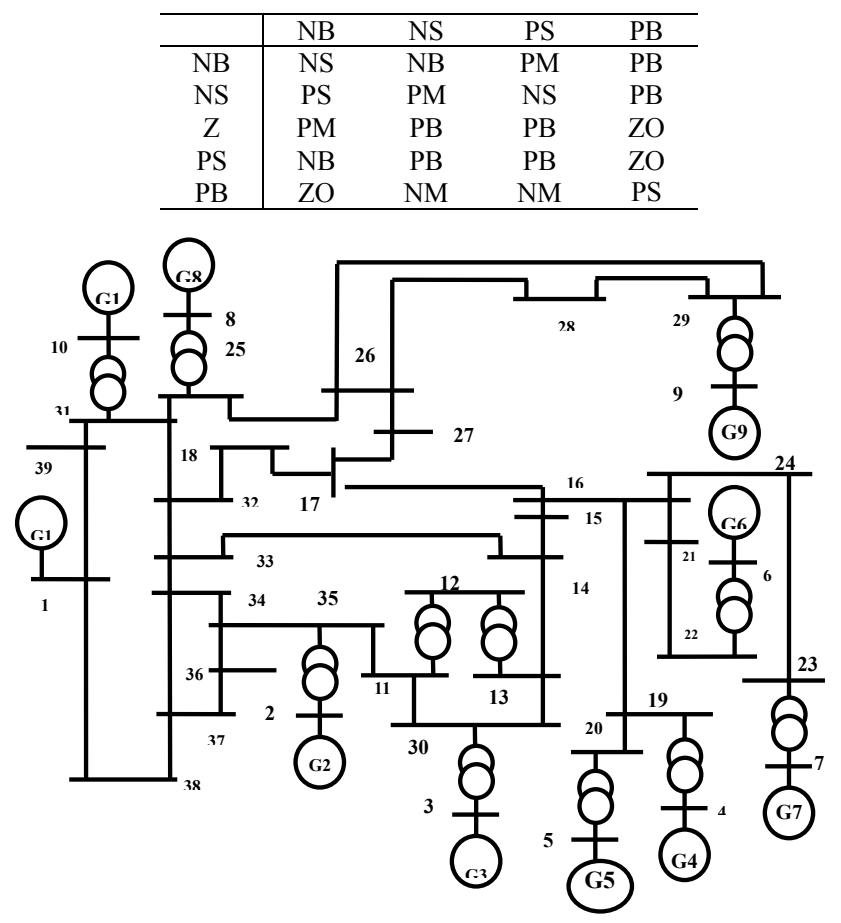

Figure 6. Ten-machine 39-bus New England power system

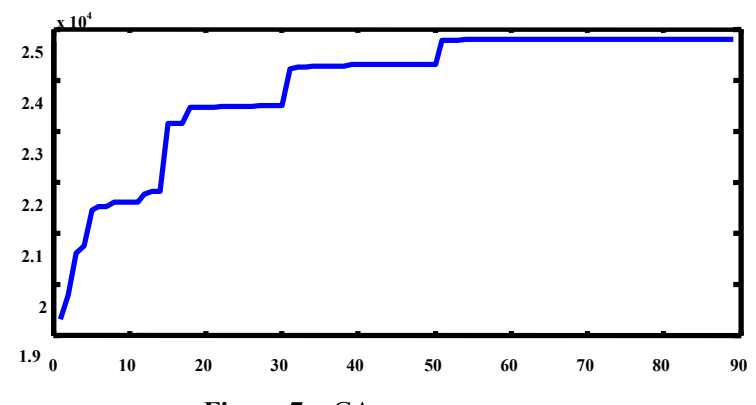

Figure 7. GA convergence

Table 3. Optimal rule base of $\mathrm{K}_{\mathrm{li}}$

\begin{tabular}{c|cccc}
\hline & NB & NS & PS & PB \\
\hline NB & NS & NB & PM & PB \\
NS & PS & PM & NS & PB \\
Z & PM & PB & PB & ZO \\
PS & NB & PB & PB & ZO \\
PB & ZO & NM & NM & PS \\
\hline
\end{tabular}

Table 4. Optimal rule base of $\mathrm{K}_{\mathrm{di}}$

\begin{tabular}{c|cccc}
\hline & NB & NS & PS & PB \\
\hline NB & PM & PB & ZO & PB \\
NS & ZO & NB & NB & NB \\
$Z$ & PS & NB & NB & NS \\
PS & NM & NB & NB & NB \\
PB & NS & ZO & PM & NM \\
\hline
\end{tabular}

\subsection{Simulation Results}

To demonstrate the effectiveness and robustness of the proposed GA based fuzzy PSS, simulation studies are carried out under fault disturbances for three scenarios. The performance of the proposed PSSs is compared to the PSSs which tuned using the Tabu Search (TS) method[12] for different operating conditions.

\section{A. Scenario 1}

In this scenario, performance of the proposed stabilizer under transient conditions is verified by applying a 6-cycle three-phase fault at $\mathrm{t}=1 \mathrm{sec}$, on bus 25 at the end of line 25-26. The fault is cleared by permanent tripping the faulted line. Speed deviations of the generators $\mathrm{G}_{5}, \mathrm{G}_{7}$ and $\mathrm{G}_{9}$ under nominal and light load condition are shown in Figs. 8-9, respectively. It can be seen that the overshoot, undershoot, settling time and speed deviations of all machines are greatly reduced by applying the proposed GA based fuzzy PSSs.

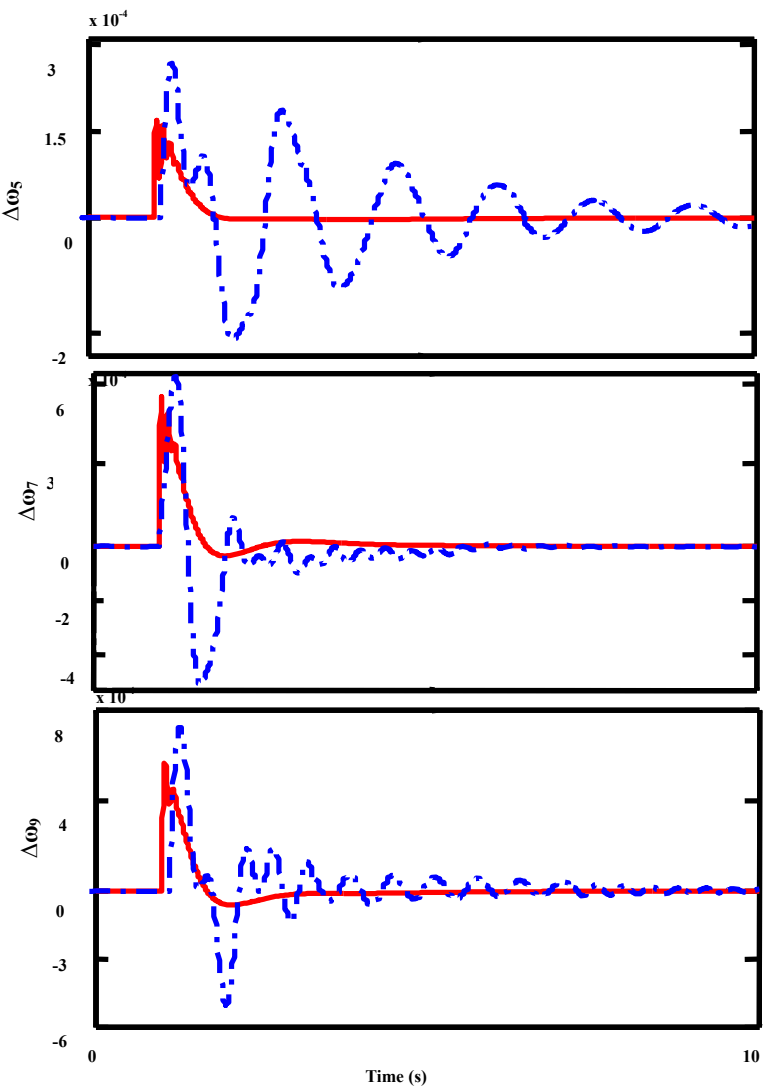

Figure 8. System response under scenario 1 with nominal loading condition: Solid (GA-F PSS) Dashed (TSPSS)

\section{B. Scenario 2}

In this scenario a 0.2 p.u. step increase in mechanical torque was applied at $\mathrm{t}=0.5$ and after a few seconds a 6 -cycle three-phase fault at $\mathrm{t}=5 \mathrm{sec}$, on bus 26 at the end of line 
26-29 for system will be applied. The results of simulation in light and heavy load condition are shown in Figs. 10-11. It can be seen that the overshoot, undershoot, settling time and speed deviations of all machines are greatly reduced by applying the proposed GA based fuzzy PSSs.

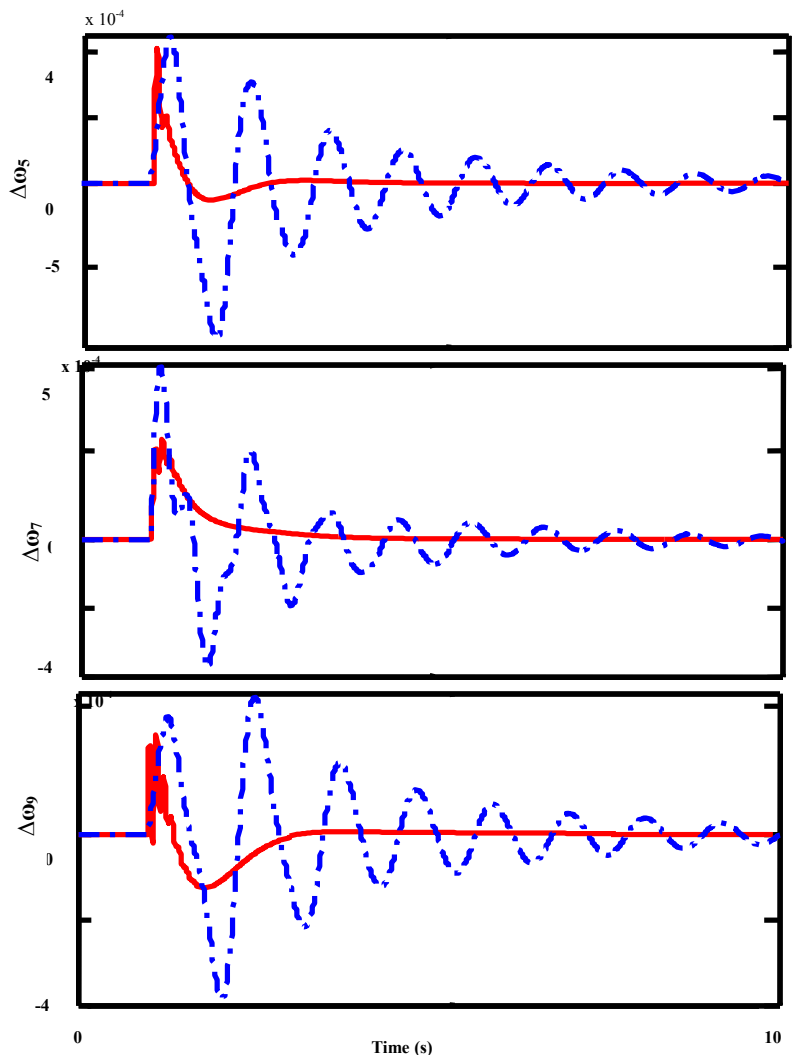

Figure 9. System response under scenario 1 with light loading condition Solid (GA-FPSS) Dashed (TSPSS)
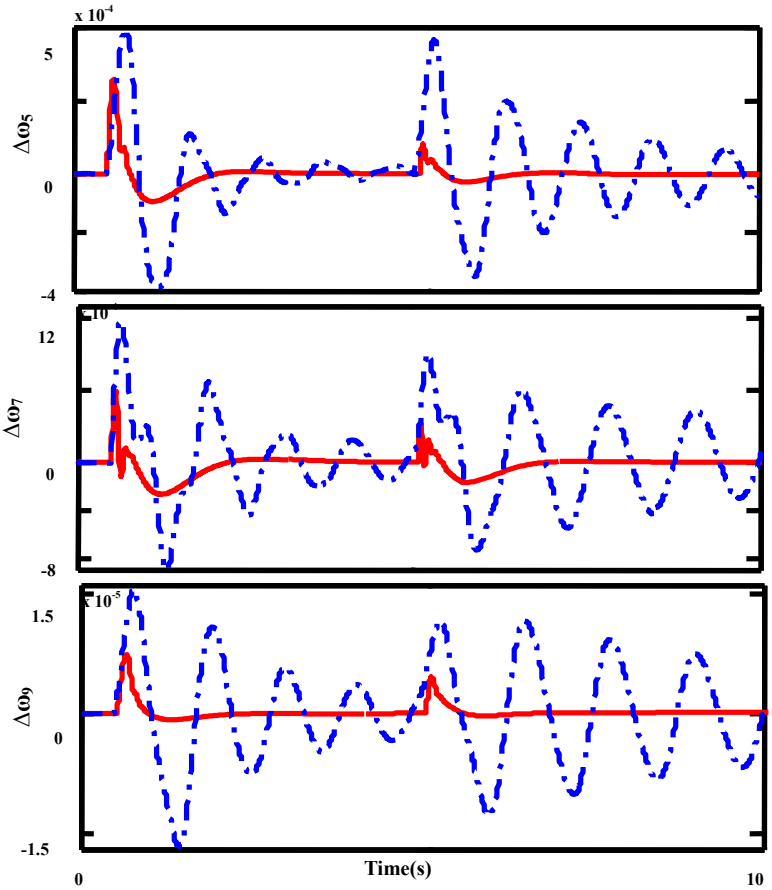

Figure 10. System response under scenario 2 with light loading condition: Solid (GA-F PSS) Dashed (TSPSS)

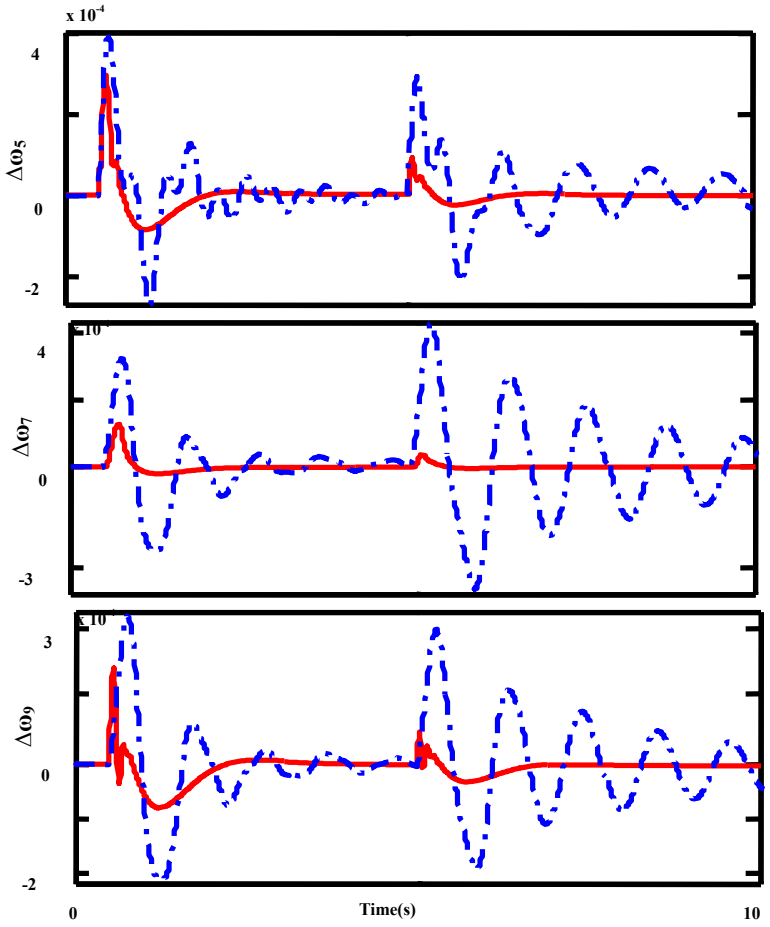

Figure 11. System response under scenario 2 with heavy loading condition: Solid (GA-F PSS) Dashed (TSPSS).

To demonstrate performance robustness of the proposed method, two performance indices: the Integral of the Time multiplied Absolute value of the Error (ITAE) and Figure of Demerit (FD) based on the system performance characteristics are defined as[15]:

$$
\begin{gathered}
I T A E_{i}=100 \int_{0}^{10} t\left(\left|\Delta \omega_{i}\right|\right) d t \\
F D=\left(O S \times 10^{4}\right)^{2}+\left(U S \times 10^{4}\right)^{2}+T_{s}{ }^{2}
\end{gathered}
$$

Where, Overshoot (OS), Undershoot (US) and settling time of rotor angle deviation of one machine is considered for evaluation of the FD. It is worth mentioning that the lower the value of these indices is, the better the system response in terms of time-domain characteristics. Numerical results of performance robustness for all cases are listed in Tables 5-6. It can be seen that the values of these system performance characteristics with the proposed controller are much smaller compared to that TSPSS. This demonstrates that the overshoot, undershoot settling time and speed deviations of all machines are greatly reduced by applying the proposed GA based fuzzy PSSs.

Table 5. Value of ITAE with different methods

\begin{tabular}{c|c|ccc|}
\multicolumn{2}{c}{ Method } & Nominal & Light & Heavy \\
\hline Scenario & TSPSS & 103.2 & 95.6 & 114.3 \\
1 & GA-FPSS & 4.31 & 3.89 & 4.91 \\
\hline Scenario & TSPSS & 228 & 207.4 & 257.6 \\
2 & GA-FPSS & 8.72 & 7.83 & 9.25 \\
\hline
\end{tabular}


Table 6. Value of FD with different methods

\begin{tabular}{c|c|ccc}
\multicolumn{2}{c}{ Method } & Nominal & Light & Heavy \\
\hline \multirow{2}{*}{ Scenario 1 } & TSPSS & 627 & 618 & 716 \\
& GA-FPSS & 109 & 101 & 117 \\
\hline \multirow{2}{*}{ Scenario 2 } & TSPSS & 759 & 735 & 776 \\
& GA-FPSS & 175 & 163 & 201 \\
\hline
\end{tabular}

From the Table 5 it can be seen that using the performance GA based fuzzy PSS in scenario 2 the ITAE index is improved about 30 times than the TSPSS one. Also the FD index is enhanced about $\% 400$ using the proposed stabilizer than the TSPSS.

\section{Conclusions}

A GA based fuzzy PSS is proposed for solution of the low frequency oscillation problem in a multi-machine power system in this paper. This newly developed control strategy combines advantage of the fuzzy control system and GA techniques for achieving the desired level of robust performance under different operating conditions and load disturbances. It should be noted that the construction of the optimal rule base sets for the proposed fuzzy PSS is very important to achieve the best performance. Thus, to reduce the fuzzy system effort and cost saving, a modified GA has been used to produce fuzzy rule Tables. The salient feature of the proposed method is that it does not require an accurate model of the system. All PSSs are designed simult-aneously, by taking into consideration the interaction among them. The effectiveness of the proposed method is tested on 10 machine 39 buses power system for a wide range of load demands and disturbances under different operating conditions. The nonlinear time simulation results confirm that the proposed FPSS can work effectively over a wide range of loading conditions and is superior to the classical Tabu search based tuned PSS.

\section{APPENDIX}

\section{Machine Models Parameters}

$\begin{array}{rr}\delta & \text { Rotor angle } \\ \omega & \text { Rotor speed } \\ P_{m} & \text { Mechanical input power } \\ P_{e} & \text { Electrical output power } \\ E_{q}^{\prime} & \text { Internal voltage behind } x_{d}^{\prime} \\ E_{f d} & \text { Equivalent excitation voltage } \\ T_{e} & \text { Electic torque } \\ T_{d o}^{\prime} & \text { Time constant of excitation circuit } \\ K_{A} & \text { Regulator gain } \\ T_{A} & \text { Regulator time constant } \\ v_{r e f} & \text { Reference voltage } \\ v & \text { Terminal voltage }\end{array}$

\section{REFERENCES}

[1] A. Safari, R. Jahani, M. Hajinasiri, A.H. Araskalaei and H.A. Shayanfar, "COA based coordinated design of FACTs and PSS output feedback controllers", International Review of Electrical Engineering, vol. 21, no. 6, 2010.

[2] K. R. Padiyar, "Power System Dynamics, Stability and Control”, Second Ed., 2006.

[3] J. H. Hwang, D. W. Kim, J. H. Lee and Y. J. An, "Design of fuzzy power system using adaptive evolutionary algorithm", Engineering Applications of Artificial Intelligence, vol. 21, pp. 86-96, 2008.

[4] H. Shayeghi, A. Jalili and H. A. Shayanfar, "Multi-Stage Fuzzy Load Frequency Control Using PSO”, Energy Conversion and Management, vol. 49, pp. 2570-2580, 2008

[5] A. L. Barreiros, M. D. Ferreira, T. Costa, Jr, W. Barre and Jr. A. P. Lopes, "A neural power system stabilizer trained using local linear controllers in a gain-scheduling scheme, " Electrical Power Energy Systems, vol. 27, pp. 473-479, 2005.

[6] M. Caner, N. Umurkan, S. Tokat and S. V. Ustun. "Determination of optimal hierarchical fuzzy controller parameters according to loading condition with ANN", Expert systems with applications, Vol. 34, pp. 2650-2655, 2008.

[7] Z. Barto, "Robust control in a multimachine power system using adaptive neuro-fuzzy stabilizers", IEE Proceedings on Generation, Transmission and Distribution, vol. 151, No 2, pp. 261-267, 2004.

[8] R. Segal, A. Sharma and M. L. Kothari,. "A self-tuning power system stabilizer based on artificial neural network", Electrical Power and Energy Systems, vol. 26, pp. 423-430, 2004.

[9] H. A. Shayanfar, H. Shayeghi, O. Abedinia and A. Jalili, "Design rule-base of fuzzy controller in multi-machine power system stabilizer using genetic algorithm", In: Proceedings of the international conference on artificial intelligence, Las Vegas, Nevada, USA, pp. 43-49, 2010.

[10] H. Shayeghi, H. A. Shayanfar, A. Safari and R. Aghmasheh, "A robust PSSs design using PSO in a multi-machine environment. Energy Conversion and Management; vol. 51, pp. 1656-1670, 2010.

[11] H. Shayeghi, H. A. Shayanfar, A. Jalili, "Multi-stage fuzzy PID load frequency controller in a restructured power system", Journal of Electrical Engineering, vol. 58, No. 2, pp. 61-67, 2007.

[12] M. Eslami, H. Shareef, A. Mohamed and M. Khajehzadeh, "Damping of power system oscillations using genetic algorithm and particle swarm optimization", International Review of Electrical Engineering, vol. 5, No. 6, pp. 2745-2753, 2010.

[13] A. Visioli, "Tuning of PID controllers with fuzzy logic". Proceeding of the IEEE International Conference on Control Theory and Applications, vol. 4, No. 1, pp. 1-8, 2001.

[14] F. Herrera, J. L. Verdegay, "Genetic algorithms and soft computing”, Physica-Verlag Heidelberg; 1996.

[15] H. Shayeghi, A. Jalili and H. A. Shayanfar, "Robust modified GA based multi-stage fuzzy LFC", Energy Conversion and Management, vol. 48, 2007, pp. 1656-1670.

[16] F. Cupertino, V. Giordano, D. Naso, L. Salvatoro, B. Tur- 
chiano, "Optimization of fuzzy controllers for industrial manipulators via genetic algorithms", Proceedings of the Industrial Electronics Conference, vol. 1, No. 6. PP. 460-465, 2003.

[17] S. Duman and A. Öztürk, "Robust design of PID controller for power system stabilization by using real coded genetic algorithm", International Review of Electrical Engineering, vol. 5, n. 5, pp. 2159-2170, 2010.
[18] Y. P. Kuo and Li. THS, "GA-based fuzzy PI/PD controller for automotive active suspension system", IEEE Trans. on Industrial Electronics, vol. 46, No. 6, pp. 1051-1056, 1999.

[19] J. Barati, S. S. Mortazavi and A. Saidian, "Analysis and assessment of FACTS-based stabilizers for damping power system oscillations using genetic algorithms", International Review of Electrical Engineering, vol. 5, No. 6, pp. 2819-2827, 2010. 